\title{
Serum Lactate Dehydrogenase as a Predictor of Outcome in Posterior Reversible Encephalopathy Syndrome: Imperative to Unify
}

W e read with great interest the study of Junewar et $\mathrm{al}^{1}$ in the September issue of the American Journal of Neuroradiology, which justified the statements that higher serum lactate dehydrogenase $(\mathrm{LDH})$ levels were possible predictors of poor outcome $(P=.009)$ but were not correlated with the severity of posterior reversible encephalopathy syndrome (PRES). Prior small series investigating the relationship between $\mathrm{LDH}$ and PRES have shown elevation of serum LDH at the time of symptom onset in patients with a variety of underlying conditions. ${ }^{2}$ Elevation of LDH levels has also been reported to precede the development of brain edema in pre-eclampsia/eclampsia by several days. Variable degrees of edema expression in PRES may be related to the variance of arterial anatomy, pre-existing disease processes, or an underlying clinical toxic condition. Hypoxia could induce tissue morphologic change and LDH increase, which is likely to result in microcirculation disturbance, followed by either enhanced vascular permeability, vasoconstriction with altered intrinsic vascular tone from platelet aggregation, or inflammatory cytokine expression. In fact, elevated serum LDH levels should also be regarded as an early biochemical marker of PRES, which has potential practical value in predicting the degree of brain edema in patients with eclampsia or cancer chemotherapy or in those receiving immunosuppression therapy in clinical practice. ${ }^{3}$

Although the underlying pathophysiologic mechanism behind edema development needs further determination, a unified standard evaluation method of brain edema severity should be adopted in a quantitative PRES study. We confirmed that the serum LDH level correlates with the extent of vasogenic edema in PRES. The overall severity of the systemic process might be reflected by the degree of edema expression in PRES. ${ }^{4}$ Junewar et $\mathrm{al}^{1}$ emphasized that the absolute values of LDH might not correlate with either the degree of cerebral endothelial dysfunction or the severity of cerebral edema. They might have confused the difference between the severity and extent of edema. In their study, the severity of PRES was classified on the basis of the extent of the hyperintensity by using FLAIR imaging, mass effect, and signs of herniation and involvement of atypical locations. Several methods of grading brain edema in PRES have been established in the

http://dx.doi.org/10.3174/ajnr.A4243 past decade. In most situations, the extent of hyperintensities in each region was graded on a scale of $0-3$ ( 0 , normal-appearing brain parenchyma; 1 , subtle signal abnormalities that are only faintly visible; 2 , large confluent areas of hyperintensity abnormalities that are easily perceptible; and 3, complete involvement of brain region) or on a scale of $1-5$ ( 1 , limited cortex white matter edema; 2 , white matter cortex edema with some deep white matter extension; 3 , white matter cortex edema with limited ventricle surface extension; 4 , white matter cortex edema, diffuse, widely confluent, extensive ventricle contact; 5 , severe white matter cortex edema, diffuse confluence, ventricle deformity). A similar edema grading method integrating edema location and distributional extent was adopted in our study, which avoided the disadvantage of simply relying on the most severely involved region of the visualized hemispheric PRES pattern. ${ }^{4}$

In our study, the extent and severity of brain edema were graded by 2 observers blinded to patients' clinical data (blood pressure, laboratory test). ${ }^{4} \mathrm{On}$ the basis of the anatomic distribution on FLAIR images with scored 1 in any regions, the distributional locations were divided into frontal, temporal, parietal, occipital, cerebellum, brain stem, basal ganglia, deep white matter, and corpus callosum. Any difference in interpreting images was settled by consensus, which would objectively reduce observational bias from different raters and improve the reliability of the results. ${ }^{4}$ On the other hand, because nonconformity exists in the description of edema distribution and radiologic features among different interpreters, a consistency test (such as a $\kappa$ test) should be performed among them. In one of the largest clinical series of patients with PRES from the Mayo Clinic, radiologic evaluation was completed by 2 neuroradiologists independently blinded to patients' clinical details. ${ }^{5}$ The frequency of agreement on imaging features differed markedly in these 2 reviewers. The most consistent characteristics established were subcortical involvement and the presence of intracranial hemorrhage (in agreement for $98 \%$ of cases in each). The presence of restricted diffusion was resolved in $79 \%$ of cases; edema severity and asymmetry were both resolved in only $63 \%$ of cases. Therefore, it is necessary to adequately define a unified scoring criteria for evaluating the severity of brain edema and enhancing the comparability among multiple studies. The time of serum sampling and the cutoff level of LDH increase 
should also be considered as another confounding factor interfering with the results.

In conclusion, a unified edema grading and serum LDH testing should be established to bring more convincing results and to standardize future correlations objectively. The role of clinical and biochemical parameters in predicting the prognosis of patients with PRES warrants further verification.

\section{REFERENCES}

1. Junewar V, Verma R, Sankhwar PL, et al. Neuroimaging features and predictors of outcome in eclamptic encephalopathy: a prospective observational study. AJNR Am J Neuroradiol 2014;35:1728-34

2. Fitzgerald RT, Wright SM, Samant RS, et al. Elevation of serum lactate dehydrogenase at posterior reversible encephalopathy syndrome onset in chemotherapy-treated cancer patients. J Clin Neurosci 2014;21:1575-78
3. Gao B. Serum lactate dehydrogenase as an early marker of posterior reversible encephalopathy syndrome. Anaesth Intensive Care 2012; 40:902-03

4. Gao B, Liu FL, Zhao B. Association of degree and type of edema in posterior reversible encephalopathy syndrome with serum lactate dehydrogenase level: initial experience. Eur J Radiol 2012;81: 2844-47

5. Fugate JE, Claassen DO, Cloft HJ, et al. Posterior reversible encephalopathy syndrome: associated clinical and radiologic findings. Mayo Clin Proc 2010;85:427-32

C. Lv

Department of Neurology

Yantai City Yantaishan Hospital and Yantai Sino-France Friendship Hospital Yantai, Shandong Province, China B. Gao

Department of Radiology Yantai Yuhuangding Hospital, Qingdao University Yantai, Shandong Province, China 